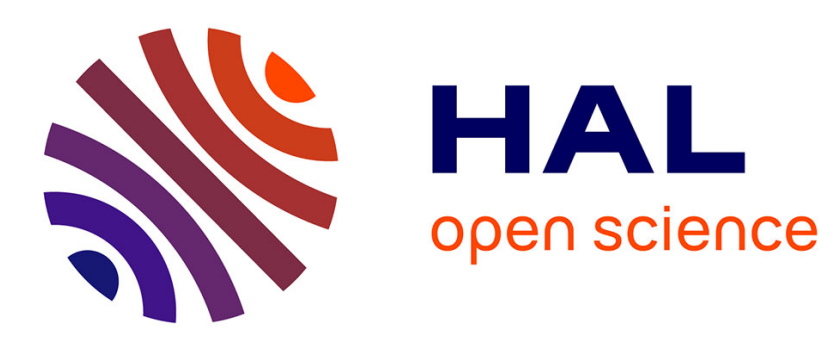

\title{
Use of probabilistic relational model (PRM) for dependability analysis of complex systems
}

Gabriela Medina-Oliva, Philippe Weber, Eric Levrat, Benoît Iung

\section{To cite this version:}

Gabriela Medina-Oliva, Philippe Weber, Eric Levrat, Benoît Iung. Use of probabilistic relational model (PRM) for dependability analysis of complex systems. 12th IFAC Symposium on Large Scale Systems: Theory and Applications, LSS 2010, Jul 2010, Villeneuve d'Ascq, France. pp.CDROM. hal-00549877

\section{HAL Id: hal-00549877 \\ https://hal.science/hal-00549877}

Submitted on 22 Dec 2010

HAL is a multi-disciplinary open access archive for the deposit and dissemination of scientific research documents, whether they are published or not. The documents may come from teaching and research institutions in France or abroad, or from public or private research centers.
L'archive ouverte pluridisciplinaire HAL, est destinée au dépôt et à la diffusion de documents scientifiques de niveau recherche, publiés ou non, émanant des établissements d'enseignement et de recherche français ou étrangers, des laboratoires publics ou privés. 


\title{
Use of Probabilistic Relational Model (PRM) for Dependability Analysis of Complex Systems
}

\author{
G. Medina Oliva, P. Weber, E. Levrat, B. Iung
}

CRAN, Nancy-Université, CNRS, Boulevard des Aiguillettes B.P. 70239 F-54506 Vandouvre lès Nancy (e-mail: \{gabriela.medina-oliva, benoit.iung,eric.levrat\}@cran.uhp-nancy.fr,\{philippe.weber\}@esstin.uhp-nancy.fr

\begin{abstract}
This paper proposes a methodology to develop a decision-making aid tool which purpose is to assess the dependability and performances of an industrial system. This model is based on a new formalism, called the probabilistic relational model (PRM) which is adapted to deal with large and complex systems. The objective is to evaluate system's performances in order to optimize the enterprise maintenance strategies. The methodology is formalized from functional, dysfunctional and informational studies of the technical industrial systems. This methodology is applied, for modeling a water heater system to estimate its performances (i.e. reliability).
\end{abstract}

Keywords: Probabilistic relational model, maintenance, system performances

\section{INTRODUCTION}

Nowadays high competitiveness makes that enterprises search higher performances at lower costs. To achieve this goal it is necessary to optimize the operations of the industrial system (system of interest) and its support systems such as the maintenance system. As a consequence, the importance of the maintenance function has increased, due to its role in improving availability, performance efficiency, products quality, on-time deliveries, environment and safety requirements, and total plant cost effectiveness at high levels (Alsyouf, I. 2007). In order to improve business performances, maintenance is thus directly related to risk analysis and dependability which allow to forecast the gaps between nominal and non-nominal operations of the system (degradation, failure, etc).

There are several works in the maintenance, however, it is somewhat "under-developed" " (McKone et al., 2001) with a lack of effective methodologies and the integration of standard methods in manufacturing companies. Maintenance is composed of a set of activities for which it is difficult to find procedures and information support systems in one place to ease the improvement process (Crespo, 2007). For this reason, our goal is to develop a methodology taking into account different conflicting criterion such as availability, safety and costs, etc., (Kiker et al., 2005) in a single model in order to optimize maintenance strategies. To face this challenge, a first step is to take into account the interrelations between the system of interest (such as the production one) and its support system (in this case the maintenance one) to assess performances. This paper explains the main concepts of an approach to formalize a model required to evaluate risk analysis, maintenance and dependability. The originality of this formalization is to unify multiple and different kinds of knowledge in one model.

\section{PROBLEM STATEMENT}

Our challenge is to formalize a generic model of complex systems to evaluate maintenance strategies. It allows to assess the global performance integrating various dimensions such as technical, organizational, human, informational, decisional and financial dimensions correlated with system's behavior and nowadays environmental factors which are necessary to quantify failure scenarios and risky situations. Furthermore, while modeling these factors, it is required to take into account the knowledge integration of diverse natures such as qualitative (organizational and human analyses) and quantitative (technical analyses) knowledge with several abstraction levels (Muller, 2007).

In that way, to estimate and to improve performances such as the reliability and safety of systems, Boudali and Dugan (2005b), Langseth and Portinale (2007) show the increasing interest on the use of Bayesian Networks. Some of their benefits are that they allow: to model complex systems, to make predictions as well as diagnostics, to compute exactly the occurrence probability of an event, to update the calculations according to evidences and to represent multimodal variables. Nevertheless, one of the scientific issues of this method is their limitation by the number of variables used in a model (Koller and Pfeffer, 1998).

Thus, our proposal consists mainly in formalizing a methodology to define an extension of Object Oriented Bayesian Networks called the Probabilistic Relational Model (Koller and Pfeffer, 1998) to model technical knowledge of an industrial system in order to help decision-making for risk, maintenance and dependability domains. The network is not defined by a graph but in a language. The proposed methodology has originality on formalizing, by means of PRM, the models from prior knowledge on the primary system functioning, malfunctioning and informational point of view to estimate the overall performance of the process (reliability and compliance of output flows).

The idea is to formalize the interactions between an industrial system (system of interest) and the support system (such as the maintenance system) using processing and data models such as SADT, AMDEC, HAZOP, etc. (Figure 1). From the 
formalized knowledge, it is necessary to establish a coding semantic with the required knowledge and programming rules to obtain a generic maintenance model which enables to predict or diagnose the impact of the influencing factors on the system global performances.

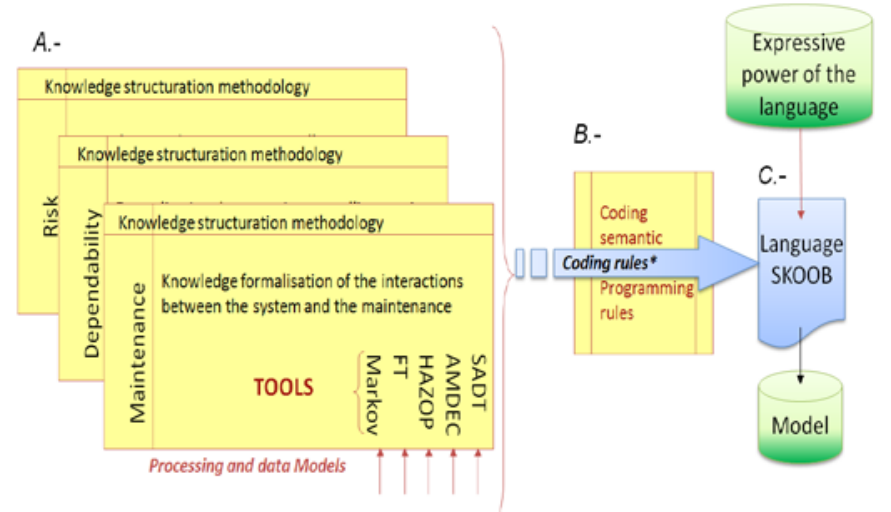

Figure 1: Methodology to formalize knowledge within a PRM

This research work is developed within the SKOOB project (http://skoob.lip6.fr/doku.php?id=public:texte_anglais_de_pr esentation) sponsored by the French National Research Agency. This project focuses on the development of a generic model based on PRM (Getoor et al., 2007) which enables to solve the formalism of complex models in risk analysis, maintenance and dependability, applied to various socioeconomic systems of strategic importance (nuclear, food industries, medical or social organizations).

\section{3.- PROPOSITION OF PRM APPROACH}

As discussed in the section 2, bayesian networks (BN) appear to be a solution to model complex systems in our domain of interest (Medina-Oliva et al., 2009). Nonetheless, one of the weak points is that $\mathrm{BN}$ are not adequate for dealing with very large complex systems. The Object-Oriented Bayesian Networks (OOBNs) extended the language of BNs with these additional concepts (Weber and Jouffe, 2006). However, the language of OOBNs is quite restricted, in a way they are not able to represent arbitrary relations between objects and uncertainty over system structure which is necessary in our domains (Pfeffer et al., 1999).

An extension of the OOBNs, the Probabilistic Relational Model (PRM) formalism allows to model real-world situations. This language is able to represent the notion of an object and the notion of a class of objects that captures properties common to an entire set of similar objects, to inherit attributes and behaviors of classes, to take advantage of the aggregation properties of the set of objects through quantifier attributes, to formalize important types of uncertainty that cannot be accommodated within the framework of traditional BN or OOBN: uncertainty over the set of entities present in a model, and uncertainty about the relationships between these entities (Koller and Pfeffer, 1998). Another advantage of the PRM is their inference aspect. Actually, it is possible to make queries so the inference is made in a specific compiled part of the model. It is not necessary to calculate the whole model that is never built but that is described in a language. (Pfeffer et al., 1999; Getoor et al., 2007 ):

To create a generic, complex and big size model to evaluate risk, maintenance and dependability it is essential to use a compact representation of knowledge (Figure 1a). To assess the influencing factors in these big size models, the PRM seems to be an efficient solution since the knowledge must be divided into groups of "local knowledge", that could be added according to the needs in order to form a whole coherent model (Figure 1c).

\section{KNOWLEDGE FORMALISATION}

The proposed modeling approach consists, from functioning systemic analysis, (a) in representing the abnormal operation (malfunctioning) (Muller, 2007), (Weber P., Jouffe L. 2006), (b) in representing the informational point of view and then (c) in formalizing and unifying these results in a unique model by means of the language based on PRM (Figure 1b).

\section{System functioning modeling}

The functional modeling of an industrial system consists in formalizing, by qualitative causal relationships, the interactions between the functions performed by each of the sub-systems until the component level (elementary functions). This type of formalization can be supported by a method such as the Structured Analysis and Design Technique (SADT).

The system functioning modeling is based on the principle of activity and sub-activities until elementary activities, supported by components, are emerging; plus the notions of system's theory (Mayer, 1996). Each activity (Figure 2) fulfils finality, which is to modify a "product" carried out by the manufacturing system. It produces or consumes flows such as "Having to Do" (HD) materializing the Input/Output (I/O) finality, "Knowing How to Do" (KHD) materializing the I/O knowledge, "being Able to Do" (AD) representing I/O energies, resources, activity support and finally "Wanting to Do” (WD) materializing the I/O triggers.

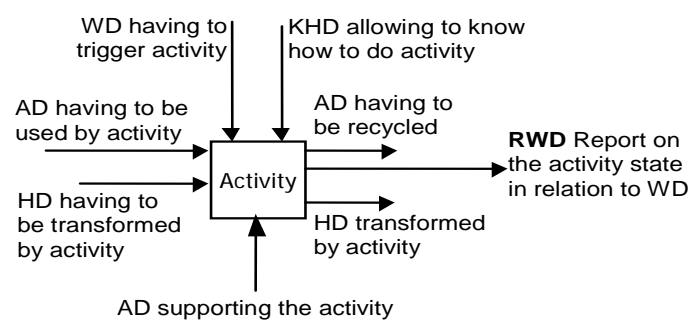

Figure 2: Flows and Activity Representation

For example, the output flow WD is a report (RWD) that represents the informational result of the Input HD product flow transformed by the activity.

\section{System Malfunctioning Modeling}

The functional model could be used to develop by duality the malfunctioning analysis, whose objectives are to identify the degraded and failure states of the components and of the 
flows, and then to determine the causes and consequences of these states on the industrial system behavior.

The degradation is spread to the rest of the system through the flow exchanging between processes, according to the causality principle:

- The potential cause of the degradation of a process is the deviation of an input flow attribute or the deterioration of its support.

- Contrarily, the potential effect of the degradation of a process is the deviation of an attribute of its output flows or its support.

The industrial system is in degradation or failure mode when there is a flow deviation and/or a deterioration of the supports of the process: the flow deviation is linked to the qualitative or quantitative deviation of a flow attribute compared to its nominal value and the support deterioration is related to the apparition of a physical mechanism of deterioration (Léger and Iung, 1998).

The dysfunctional analysis also involves the identification of groups of elementary events or combination of events that lead to a failure event, as well as, the identification of the logical links between essential components to perform the system mission.

For this aspect, there are used the following dependability methods:

- FMECA: to model failure modes of the functions, failure modes of the components, failure consequences (impact on the flow and other functions) and the criticality of the failure.

- HAZOP: to model flow deviation, cause of flow deviation and failure consequences (impact on the flow).

- $\quad$ Fault tree (FT), reliability block diagram or Bayesian networks (BN) to model the logical links of events or logical links between components.

\section{System Flow Informational Modeling}

Each flow is characterized by the state of the variables related to its morphologic, spatial or temporal properties of the objects that composed it (i.e. objects and flow of objects) and by the flow variables that are express as the quantity of objects per time unit (such as a flow rate) (Mayer, 1996). So, the state variables and the flow variables can be regroup in one denomination called flow attribute. In that way, to measure the performance of a function, it is assume the hypothesis that it can be evaluated directly from the flow attributes. The object representation allows to identify these flow properties or attributes, and it can be represented as shown on an entity-relationship diagram (Figure 3)

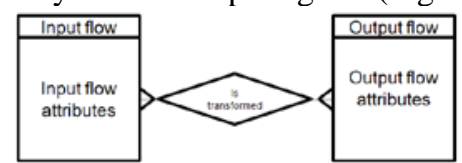

Figure 3: Extract of an entity-relationship diagram of a function and its flows

\section{Unification of Technical knowledge in a PRM model}

To model the different aspects of a system in a PRM, it is required to take into account the different types of knowledge previously identified (Figure 4).

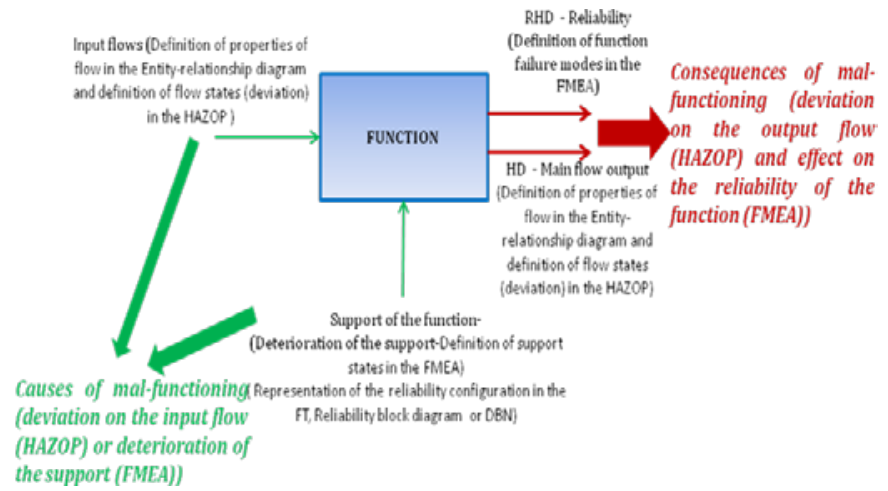

Figure 4. Technical Knowledge Formalization

To integrate this knowledge within a PRM, it is incorporated as new variables of a network or as a part of the required information to complete a conditional probabilities table (CPT) for these variables. The knowledge integration is based on the following rules (Figure 1b):

1.- Formalization of the network structure from the functional analysis (input and output variables of a process). The input and output variables are defined from the functional analysis (different kinds of input flows on the SADT) and from the informational analysis (input flow attributes on the entityrelationship diagram). Then, it is possible to compile a PRM into a Bayesian network (grounding) (Figure 5).

2.- Definition of the input and output variable states, as it is described in the malfunctioning analysis. The states of input and output variables are defined on the malfunctioning system analyses of the system, such as failure modes or flow deviations (methods FMEA or HAZOP).

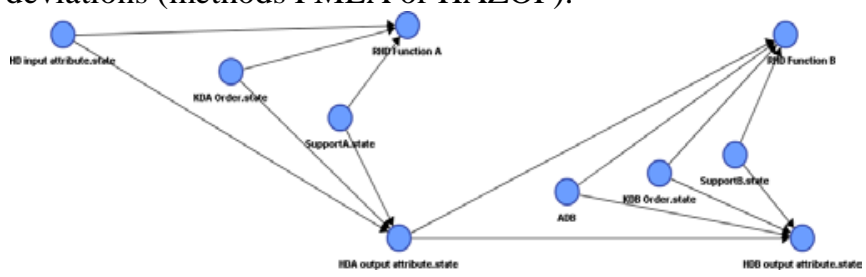

Figure 5: Network structure from functional and informational analyses

3.- Definition of the conditional probabilities given in the malfunctioning analysis (logical links between components), combinatory logic or expertise.

The conditional probabilities are related to the combinatory logic, to the frequency of failures defined on the malfunctioning analysis or to the expert's judgment. Moreover, to calculate the conditional probability of support of a function which is supported by two or more parallel components, it is possible to obtain the reliability of the support (AD support flow) of this function by means of a dynamic bayesian network, fault tree or a reliability block diagram (Figure 6).

In Figure 6 it is shown how to integrate in a CPT the variables and the conditional probabilities according to the 
different system' point of view: functioning view, malfunctioning view and the informational view.

Also, it is important to know that:

- To represent the input flow (energy, information or material flow) of a function, there could be several variables for each flow.

- $\quad$ To define an output flow, there are necessary several CPT based on the input flows. There must be one CPT for each output flow.

To integrate the different kinds of knowledge in large and complex models, the SKOOB project is developing a language to represent PRM models (Figure 1c).

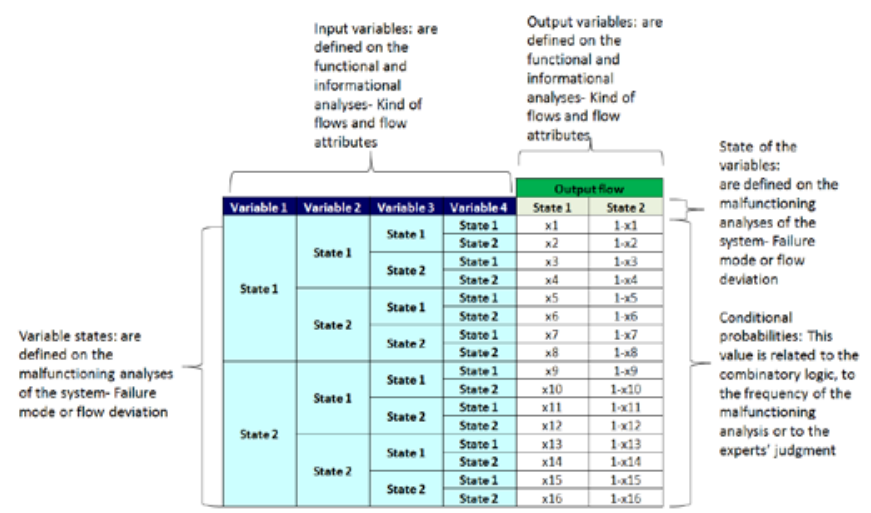

Figure 6: Knowledge integration in a CPT

This language is inspired in Java language, because it is one of the more common languages used in the present. The characteristics of SKOOB language are based on the principles of the PRM such as: compilations units like variables, models and classes (declaration of attributes, of references and of conditional probability table (CPT), specialization, quantifiers or aggregators). To illustrate the language we are developing the notion of class:

- Declaration of a class: Classes correspond to a type of entity in the domain and provide reusable probability models that can be applied to many different objects.

In the SKOOB language a class is declared by the following

way:

class Name_of_the_class \{

\}

// Body of the class

The CPT in the SKOOB language is written in the following order:

$[1.0,0.0, / / P(O K \mid$ true, $O K)$ (state $=X 1), P(O K \mid$ true, $N O K)$ (state $=X 2)$,

$0.0,1.0] / / \mathrm{P}(\mathrm{NOK} \mid$ true, $\mathrm{OK})($ state $=1-\mathrm{X} 1), P(\mathrm{NOK} \mid$ true, $\mathrm{OK})($ state $=1-\mathrm{X} 2)$

\section{APPLICATION}

To show the feasibility of the proposed knowledge formalization and the integration of the different kinds of knowledge into a PRM an application is illustrated. A classical example of a water heater process is presented in order to assess the reliability and the compliance of the output flow attributes. The objective of the thermal process (show in Figure 7) is to ensure a constant water flow rate with a given temperature. The process is composed of a tank equipped with two heating resistors R1 and R2. The system inputs are the water flow rate Qi, the water temperature $\mathrm{Ti}$ and the heater electric power $\mathrm{P}$ that is controlled by a computer. The outputs are the water flow rate Qo and the temperature $\mathrm{T}$.

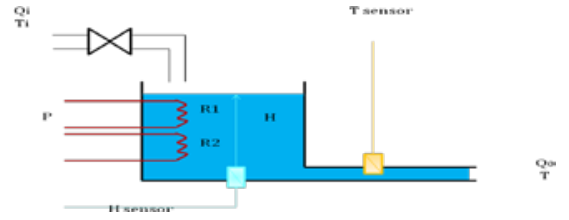

Figure 7: Water heater process

System Functioning Modeling: SADT model

Figure 8 presents the diagram A- 0 of the SADT related to the process. This figure depicts the interaction between the process and the external environment through the $\mathrm{AD}$, HD and RHD flows. The main functionality of the process is to provide warm water.

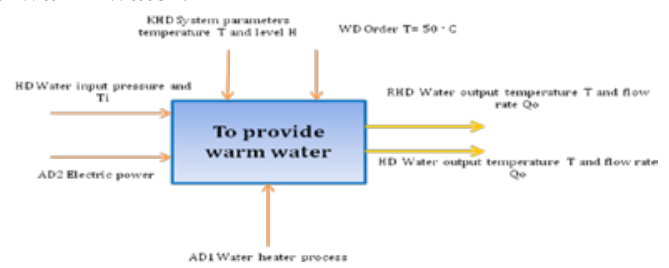

Figure 8: Diagram A-0 of the SADT

Then the diagram A0 describes the four functions that are necessary to perform the main task of the system:

- to transform pressure into Qi (A1),

- to control V and P (A2),

- to transform Qi into H and Ti into T (A3),

- to transform H into Qo (A4).

When decomposing function A3 'to transform Qi into $\mathrm{H}$ and Ti into T' one of the elementary functions is "to heat water" supported by the component HEATING RESISTOR. The input flows of the function are: $\mathrm{HD}$ storage water, $\mathrm{AD}$ electric power, WD order $\mathrm{T}$, $\mathrm{AD}$ heating resistors. The output flows are represented by the RHD water temperature $\mathrm{T}$ and the HD water temperature T.

System Malfunctioning Modelling: FMEA, HAZOP, dynamic bayesian networks.

For this case, the study will be applied to the function "to heat water", so the component of this function is indexed in the FMEA analysis (Table 1). The failure modes of the component are defined as well as their effects. The causes are linked with the component states or the unavailability of the electric energy required to supply the component.

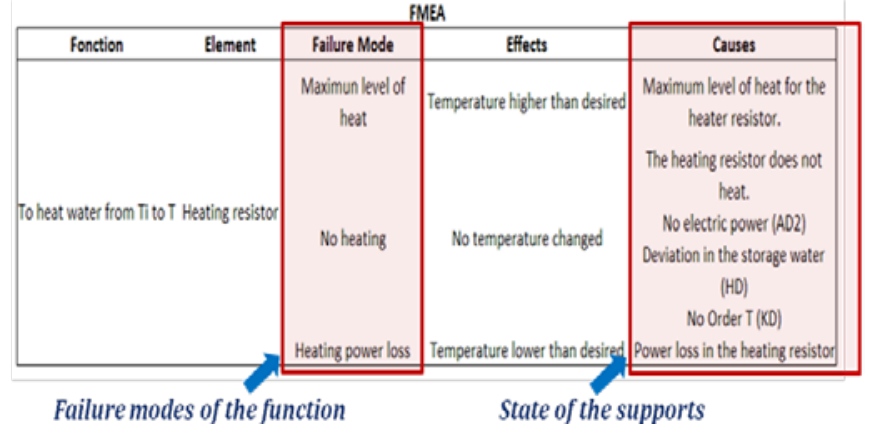

Table 1: Extract of the FMEA of the function "to heat water" 
Then, it is necessary to study the possibilities of flow deviation and their causalities through an HAZOP study (Table 2). The flow deviation is linked to the qualitative or quantitative variation of an attribute compared to its nominal value and it is a complementary study of the FMEA since the mal-functioning of an industrial system is caused when there is a flow deviation (HAZOP) and/or a deterioration of the supports of the process (FMEA).

\begin{tabular}{|c|c|c|c|c|}
\hline Products & Properties & Deviations & Causes & Consequeaces \\
\hline \multirow{5}{*}{ Storage water } & \multirow{3}{*}{ Lovel } & Mare & $\begin{array}{l}\text { H sensor is down } \\
\text { Mere rate fow of } \mathrm{OC}\end{array}$ & \begin{tabular}{|l|} 
Waxe spill \\
More outgongz flow > $>Q_{0}$
\end{tabular} \\
\hline & & Less & $\begin{array}{l}\text { H seaser is down } \\
\text { Less rate flow of Qfi } \\
\text { Leakl of wyer of tank }\end{array}$ & Less cutgoing Alow < $<0$ \\
\hline & & No & Trak is dotru & No oungsing flow Qo \\
\hline & \multirow{2}{*}{ Temperotire } & More & $\begin{array}{l}\text { T senser is down } \\
\text { Maximum kvel of heat in heas retiatsor }\end{array}$ & 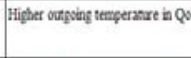 \\
\hline & & Less & $\begin{array}{l}\text { Tsenser is down } \\
\text { Heanng resistor is doun }\end{array}$ & Lower sutgong temperantre in $Q_{0}$ \\
\hline
\end{tabular}

Table 2: Extract of the HAZOP of the function "to heat water"

Since the heating resistors " $\mathrm{R} 1$ " and "R2" work on parallel to fulfill the function "to heat water", it is possible to obtain the reliability of the support (AD support flow) of this function. The state of each heating resistor was defined as follow: $80 \%$ is available, $5 \%$ works in a maximum level, $5 \%$ there is power loss in it and $10 \%$ the heating resistor is unavailable. So with this information, it is possible to build a dynamic bayesian network as shown in the Figure 9.
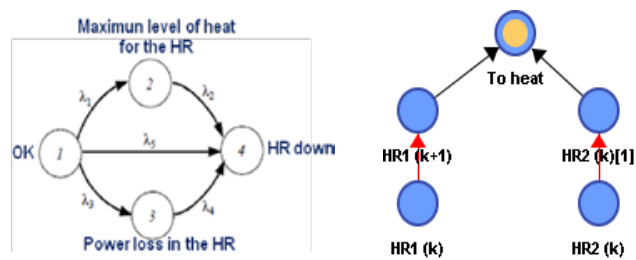

Figure 9: DBN of the parallel heating resistors

The result of support reliability of the function "to heat" shows that the AD support flow of this function is available $92 \%$ of the time, $5 \%$ works in a maximum level, $0,75 \%$ there is power loss in them and 2,25\% the heating resistors are unavailable (results obtained with the software Bayesialab). Moreover, it could be possible to use fault trees or reliability block diagrams in these cases. They can describe the logical links of events in order to obtain the reliability of the support of a function in cases where there are redundancy or $\mathrm{k} / \mathrm{n}$ relations between components. Their limitation is that they represent boolean variables, that is why it was not appropriated for this example.

\section{System Flow Informational Modeling: Entity-relationship diagram}

The informational point of view let identify the flow properties and attributes (Figure 10).

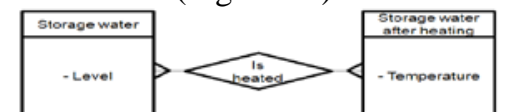

Figure 10: Extract of the entity-relationship representation of the flows of the "to heat water" function
Unification of Technical knowledge in a PRM model

Finally, it is shown the integration of the previous kinds of knowledge within the CPT of the variable: "water temperature" and its transformation into the SKOOB language (Figure 11).

This part of the CPT shows the link between the information integration available in the CPT and a part of the SKOOB language that allows to convert knowledge into a PRM

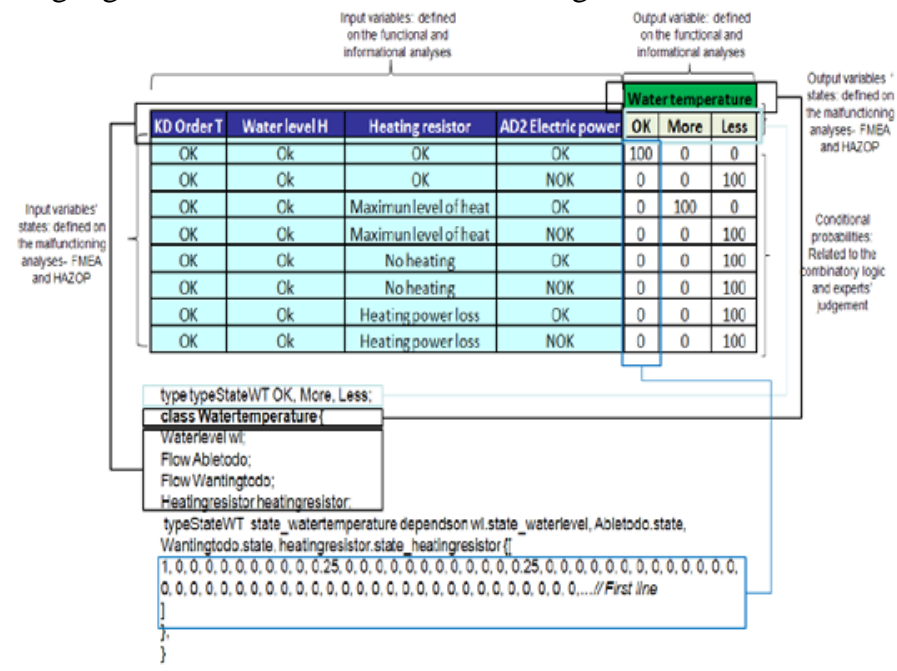

Figure 11: Knowledge integration of the variable "Water temperature" in a CPT

Finally, the SKOOB language is developed for this application. A part of it is illustrated as follow:

type typeState OK,NOK

type typeStateHR OK, Maximun_level, Power_loss, NOK; //States of the heating resistors coming from FMEA

type typeStateRHDtoheat OK, Maximun, Losspower, NOK; // States of the function "To heat water from Ti to T" coming from the FMEA

type typeStateWL OK, More, Less, No; // States of the water rate flow coming from the HAZOP

class Flow \{

boolean state $\{[1.0,0.0]\}$;

class Input_variable extends Flow \{

\} // To represent all the input variables such as $A D$ and WD.

class Abletodo extends Input_variable

\}// AD-Support flow represented by material, energy, human or software flow.

class Watingtodo extends Input_variable

\}// WD-Materialising the trigger of the function

class Waterlevel \{

typeStateWL state_waterlevel \{

// OK More Less No

$[0.85,0.05,0.05,0.05]\}$

\}//Having to Do- Main input flow of the function "To heat water from Ti to T"- The level is an attribute coming from the entity- relationship diagram / States of the water rate flow coming from the $H A Z O P$

class Heatingresistor \{

typeStateHR state_heatingresistor\{

// OK Maximun level Power loss NOK

$[0.80,0.05,0.05,0.1]\}$

\} // AD-Equipments that support the function "To heat water from Ti to T" / States of the heating resistors coming from FMEA/ Conditional probabilities coming from the DBN-

Figure 9

class RHDtoheat

Waterlevel wl;

Flow Abletodo;

Flow Wantingtodo;

Heatingresistor heatingresistor;

typeStateRHDtoheat state RHDtoheat dependson wl.state waterlevel, Abletodo.state, Wantingtodo.state, heatingresistor.state_heatingresistor $\{[$

$1,0,0,0,0,0,0,0,0,0,0.25,0,0,0,0,0,0,0,0,0,0,0,0.25,0,0,0,0,0,0,0,0,0,0,0,0$

$0,0,0,0,0,0,0,0,0,0,0,0,0,0,0,0,0,0,0,0,0,0,0,0,0,0,0,0, \ldots / /$ First line

]\}; // States of the function "To heat water from Ti to T" coming from the FMEA

IINSTANCIATON

system $\mathrm{m}\{$

//Function//

Waterlevel waterlevel;

RHDtoheat rhdtoheat; //elementary function of the system // 
// Definition of all input flows of the function "To heat water"//

rhdtoheat.wl = waterlevel;

Input_variable OrderT:

rhdtoheat Wantingtodo $=$ OrderT

Heatingresistor heatingresistor;

rhdtoheat.heatingresistor = heatingresistor;

Abletodo electricpower;

rhdtoheat.Abletodo = electricpower;

Then, it is possible to compile a PRM into a Bayesian network (Figure 12).

\section{Use of the model}

a. As a prognosis model:

The PRM allows the analysis of the influencing flows on the functions states and on the output flow states. The objective is to forecast the impacts of input flows failures or degradations on the functions (Figure 12). There is obtained the marginal probability that the function is performed properly $(61,30 \%)$ given the prior probabilities of the input flows.

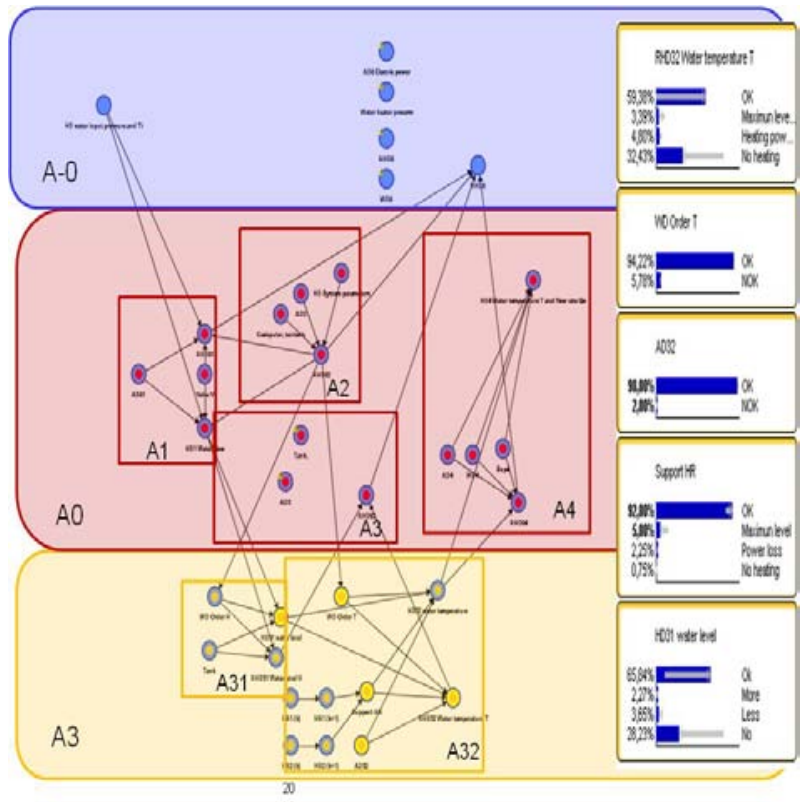

Figure 12: Prognosis in a Bayesian network for the water heater process

\section{b. As a diagnosis model:}

The diagnosis starts when the "RWD to heat water" is in abnormal functioning (state= maximum level of heating) for example. Initially, the input flows are checked to see which is the variable that has more probability of been in an abnormal functioning. For this case the water level has a probability of been in an non-nominal state of $4,03 \%$, the electric power has $0 \%$, the order $\mathrm{T} 0 \%$ and the heating resistors of $99,59 \%$. The checking leads suspect that the heating resistors are the most probable cause that the function is not realized, because its probability of been in an abnormal functioning is the highest (99,59\%).

\section{CONCLUSION AND FURTHER WORK}

The proposed model based on the functioning (SADT), malfunctioning (FMECA, HAZOP analysis, 2TBN) and informational studies (entity- relationship diagram) help to improve the automatic generation of a model to assess the reliability and the output flow compliance of a technical system.
The difference of the PRM when comparing with other classical methods is their capacity to deal with large complex domains. Also this model allow to deal with issues such as prediction or diagnostic optimization, data analysis of feedback experience, deviation detection and model updating.

However, this methodology is a first step to gather technical information in order to evaluate risk analysis, maintenance and dependability. As further work, some other factors should be incorporated in the model such as human, organizational and environmental factors. It is also necessary to manipulate uncertainties within the parameters and the knowledge of the model.

Finally, to validate the model by applying it to a real system in order to show industrial feasibility and to confirm its added value compared to the traditional computerized decisionmaking tools.

\section{AKNOWLEDGEMENT}

The authors wish to express their gratitude to the French National Research Agency ANR for the financial support of the Structuring Knowledge with Object Oriented Bayesian nets SKOOB project. Ref. ANR PROJET 07 TLOG 021 (http://skoob.lip6.fr). Special thanks are also paid to $\mathrm{P}$. Munteanu and F. Clerc from Bayesia and to P. Wuillemin and L. Torti from LIP6 who are developing the SKOOB language and the inference algorithm for the PRM.

\section{REFERENCES}

Alsyouf, I. (2007) The role of maintenance in improving companies' productivity and profitability. International Journal of Production Economics, 105, 70-78.

Boudali H., Dugan J.B. (2005b). A discrete-time Bayesian network reliability modeling and analysis framework. Reliability Engineering \& System Safety. 87(3), 337-349.

Crespo-Márquez A, (2008). The maintenance management framework: models and methods for complex systems maintenance, Springer Series in Reliability Engineering ISBN-10:1846288207.

Getoor, Lise, Nir Friedman, Daphne Koller, Avi Pfe er, and Ben Taskar. (2007). Probabilistic relational models. In Introduction to statistical relational learning Cambridge: MIT Press., chap. 5, 129-174.

Kiker,G. A., Bridges, T. S., Varghese, A., Seager, T. P.,\&Linkov, I. (2005). Application of multicriteria decision analysis in environmental decision making. Integrated Environmental Assessment and Management, 1, 95-108.

Koller D. and Pfeffer A. (1998), Probabilistic frame-based systems, Proceedings of the 15th National Conference on Artificial Intelligence (AAAI), Madison, Wisconsin..

Langseth H., Portinale L. (2007). Bayesian networks in reliability. Reliability Engineering \& System Safety. 92(1), 92-108.

Léger, J.-B. and B. Iung (1998). Methodological approach to modelling of degradation detection and failure diagnosis in complex production systems. In : 9th International Workshop on Principles of Diagnosis, 209-216, Cape Cod (USA).

Mayer, F.; Morel, G.; Iung, B.; and Léger, J-B. (1996). Integrated manufacturing system meta-modelling at the shop-floor level. In Proceedings of the Advanced Summer Institute Conference, pp. 257-264. Toulouse, France : Lab. For Automation and Robotics of Patras - Greece.

McKone KE, Schroeder RG, Cua KO, (2001) The impact of Total Productive Maintenance practices on manufacturing performance. Journal of Operations Management, 19(1):39-58.

Medina-Oliva G., Weber P., Simon C., Iung B., (2009). Bayesian Networks Applications on Dependability, Risk Analysis and Maintenance. In : 2nd IFAC Workshop on Dependable Control of Discrete Systems, 245-250, Bari, Italy

Muller A., Suhner M-C., Iung B (2007). Formalisation of a new prognosis model for supporting proactive maintenance implementation on industrial system. Reliability Engineering \& System Safety.

Pfeffer A., D. Koller, B. Milch and K.T. Takusagawa. (1999). SPOOK: A System for Probabilistic Object-Oriented Knowledge Representation. Proceedings of the 14th Annual Conference on Uncertainty in AI (UAI), Stockholm, Sweden.

Weber P., Jouffe L. (2006). Complex system reliability modelling with Dynamic Object Oriented Bayesian Networks (DOOBN). Reliability Engineering and System Safety, 91(2),149-162. 